\title{
Colpire l'infiammazione per migliorare la prognosi del paziente dializzato
}

\author{
Francesco Rastelli, Maria Carmen Luise, Giuseppe Pontoriero \\ S.C. Nefrologia e Dialisi, Ospedale "Alessandro Manzoni", Lecco
}

\begin{abstract}
Treating inflammation to improve outcome in dialysis patients
Uremia is a systemic inflammatory condition characterized by a combination of oxidative burst, uremic toxicity, uremic dyslipidemia, endothelial dysfunction and oxidative stress resulting from dysfunctional mitochondrial electron transfer generating reactive oxygen species. These compounds induce oxidative modifications of carbohydrates (AGEs), proteins, lipids and DNA, in turn recognized as DAMPs by Toll-like receptors (TLRs), which in end-stage renal disease (ESRD) are upregulated in many cell types including macrophages and neutrophils. All the inflammatory derangements typical of ESRD lead to an increased cardiovascular risk in dialysis patients. Unfortunately, the technological innovations in dialysis techniques have not given rise to a better prognosis for patients with ESRD, probably because they have had no favorable effect on inflammation in the uremic milieu. Presenting a recent review by Baragetti and coworkers published in Am J Nephrol 2017 and the results of a PubMed search with "antiinflammatory drug hemodialysis", "oxidative stress drugs hemodialysis" and "endothelial dysfunction drug hemodialysis" as keywords, we would like to offer a novel and innovative approach to targeting uremic inflammation considering data from clinical trials.
\end{abstract}

Keywords: Cardiovascular mortality, Dialysis, Endothelial dysfunction, Inflammation, Oxidative stress

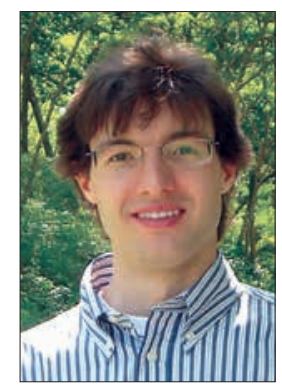

Francesco Rastelli

\section{Colpire l'infiammazione per miglio- rare la mortalità del paziente dializ- zato}

L'uremia è una sindrome infiammatoria sistemica (1) che comporta un elevato rischio cardiovascolare, in particolare per i pazienti dializzati (2-4), la cui mortalità è più alta rispetto alla popolazione generale (5). L'uremia tramite lo stress ossidativo, la disfunzione endoteliale e le tossine uremiche è per se stessa caratterizzata da infiammazione. La situazione peggiora di gran lunga se il paziente affetto da malattia renale cronica (MRC) terminale presenta una di queste tre condizioni gravate da elevata infiammazione: la sindrome metabolica,

Accepted: November 6, 2017

Published online: November 21, 2017

Indirizzo per la corrispondenza:

Dr. Giuseppe Pontoriero

S.C. Nefrologia e Dialisi

ASST Lecco, Ospedale "Alessandro Manzoni"

Via dell'Eremo, 9/11

23900 Lecco

g.pontoriero@asst-lecco.it il diabete mellito e il fumo (6). In una recente review Baragetti et al (7) tematizzano come a fronte delle innovazioni nelle tecniche dialitiche, pochi sono i progressi significativi fatti nel ridurre l'infiammazione nel paziente affetto da MRC avanzata, uno scarto inaccettabile data l'elevata mortalità cardiovascolare del paziente dializzato (3), direttamente associata allo stress ossidativo (8).

II milieu uremico è caratterizzato dal burst ossidativo (9), un processo che peggiora l'infiammazione nell'uremia. Lo stress ossidativo deriva da un alterato trasferimento di elettroni a livello dei mitocondri (10) e dalla conseguente generazione di specie reattive dell'ossigeno, i cosiddetti "radicali liberi", che inducono modificazioni ossidative dei carboidrati (Advanced glycation end products o AGEs, i prodotti di glicosilazione avanzata), delle proteine, dei lipidi e del DNA. Questi composti sono riconosciuti come "damage-associated molecular pattern molecules" (DAMPs) dai recettori Toll-like (TLRs), che sono upregulati in molte cellule nella MRC avanzata, in particolare nei macrofagi attivati di tipo $\operatorname{M1}(11,12)$ e nei monociti circolanti CD14+CD16+, linee cellulari che secernono citochine proinfiammatorie (IL-12, IL-6, IL-1b, TNF- $\alpha$ ) (11), che favoriscono la spontanea degranulazione dei neutrofili (13). La produzione di IL-1, IL-6, MCP-1 e TNF- $\alpha$ è anche aumentata dall'interazione degli AGEs col loro recettore (RAGE) via NF-kB (14). L'iperattivazione della via di segnale RAGE/AGE è associata con la progressione della MRC (15). L'espressione di NF-kB è direttamente aumentata dallo stress 
ossidativo, che provoca la degradazione della chinasi inibitoria $\mathrm{kB}(\mathrm{I} \mathrm{kB})$, una maggiore translocazione e dimerizzazione a livello nucleare di NF-kB, e un aumento della trascrizione dei geni implicati nella risposta infiammatoria (16). NF-kB è di per sé correlato alla coronaropatia negli emodializzati perché i livelli plasmatici del ligando del recettore di attivazione di NF-kB (RANKL) sono inversamente associati alle calcificazioni coronariche (17). Della review di Baragetti et al (7) prendiamo in esame i seguenti approcci antinfiammatori avvalorati da trial clinici sull'uomo:

- La supplementazione orale di vitamina E a scopo antiossidante implica una riduzione del $64 \%(R R=0,46)$ dell'incidenza di un primary endpoint combinato (infarto miocardico, stroke ischemico, vasculopatia periferica e angina instabile) e riduzione del $70 \%(R R=0,3)$ dell'incidenza di infarto miocardico in 196 pazienti emodializzati seguiti in media per 519 giorni (18).

- La supplementazione orale di pentossifillina a scopo antiossidante in 36 pazienti emodializzati per 4 mesi ha dimostrato una riduzione di TNF- $\alpha$, IL-6 e proteina $C$ reattiva (PCR) nei soggetti trattati (19).

- Anche le vitamine del gruppo B riducono il rischio di mortalità cardiovascolare nei pazienti emodializzati (20).

Novità di interesse:

- L'inibizione del complemento con anti-C1 e anti-C5a possono silenziare l'infiammazione intradialitica determinata da membrane non pienamente biocompatibili, anche se rimane una strategia costosa. Alternative più promettenti sono il peptide 5C6, PMX-53, la compstatina e POT-4 (21-23).

- La somministrazione di $100 \mathrm{mg}$ sc di anakinra per 4 setti- mane, un antagonista ricombinante del recettore umano di IL-1, riduce nei pazienti trattati in media del $53 \%$ la PCR plasmatica e del $40 \%$ i livelli della interleuchina proinfiammatoria IL-6 (24).

- Campo promettente e ultimamente molto studiato è la riduzione plasmatica grazie a probiotici intestinali di indossil-solfato e cresil-solfato (25), tossine uremiche implicate nell'adesione endoteliale di cellule infiammatorie per la maggior espressione di E-selctina e ICAM-1 (26).

- Nell'ambito della terapia cellulare, le cellule T regolatorie (Treg) (27) e le cellule mesenchimali (28) sono studiate per le possibili implicazioni nell'indurre la tolleranza immunitaria nei pazienti portatori di trapianto di rene, con la loro possibile azione antinfiammatoria nei pazienti in emodialisi cronica.

Con esito negativo o scoraggiante:

- La supplementazione di 5-metil-tetraidrofolato ha dato risultati contrastanti nel ristabilire la funzione endoteliale nei pazienti emodializzati $(29,30)$; mentre non sembra ridurre la mortalità cardiovascolare nel paziente dializzato (31).

- Etanercept, TNF- $\alpha$ antagonista, non ha prodotto un effetto antinfiammatorio su 10 dializzati trattati per 44 settimane (32).

Devono ancora essere studiati in studi clinici randomizzati (RCT) adeguati gli antagonisti di TLRs (33).

Parlare di infiammazione nell'uremia è addentrarsi in un campo molto vasto che si estende dallo stress ossidativo alla disfunzione endoteliale. Effettuando una medline su PubMed utilizzando "antinflammatory drug hemodialysis", "oxidative stress drugs hemodialysis" e "endothelial disfunction drug hemodialysis" sono emerse molte altre strategie per ridur-

TABELLA I - Strategie per ridurre l'infiammazione nel dializzato

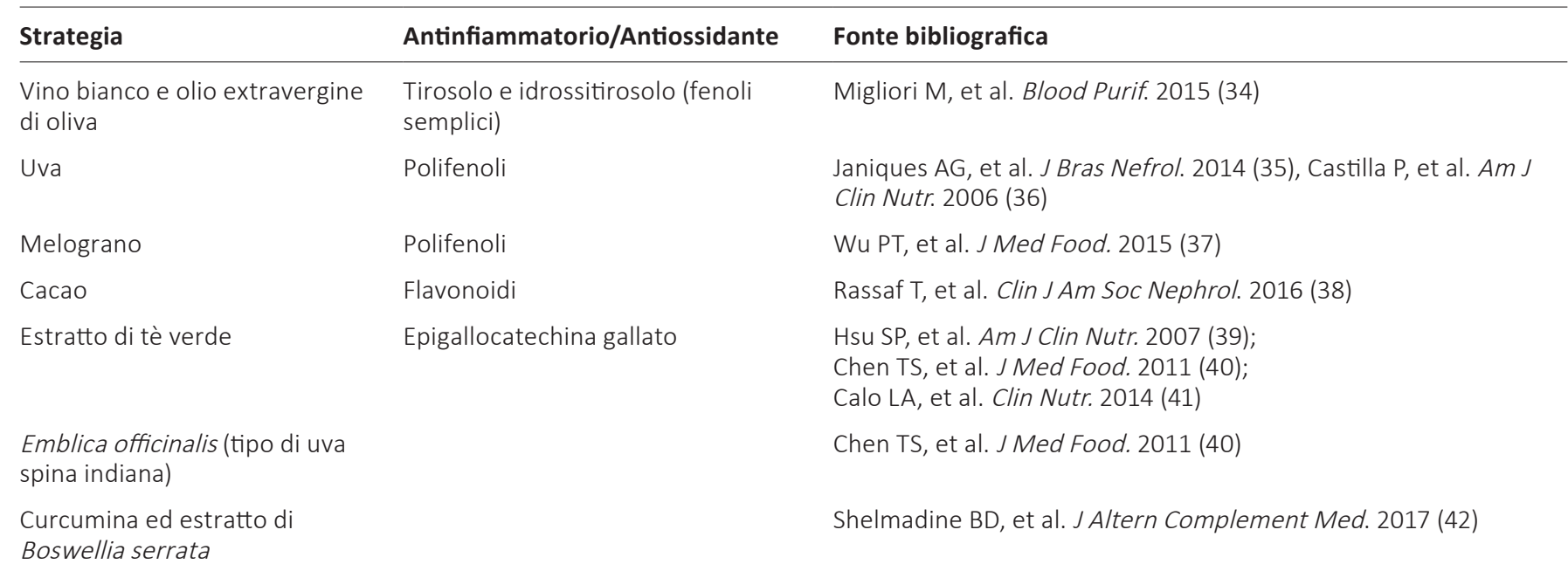


TABELLA I - Continua

\begin{tabular}{|c|c|c|}
\hline Strategia & Antinfiammatorio/Antiossidante & Fonte bibliografica \\
\hline \multirow[t]{3}{*}{ Acidi grassi polinsaturi } & Omega 3 & $\begin{array}{l}\text { Vernaglione L, et al. J Nephrol. } 2008 \text { (45); Gharekhani A, et al. Eur } \\
\text { J Clin Pharmacol. } 2014 \text { (46); } \\
\text { Moeinzadeh F, et al. Iran J Kidney Dis. } 2016 \text { (47) }\end{array}$ \\
\hline & L-carnitina & Chen Y, et al. Am J Clin Nutr. 2014 (48) \\
\hline & Febuxostat & Alshahawey M, et al. Am J Nephrol. 2017 (49) \\
\hline Fibrati & Fenofibrati & Makówka A, et al. Lipids Health Dis. 2012 (50) \\
\hline \multirow[t]{3}{*}{ Statine } & Pravastatina & Cueto-Manzano AM, et al. Arch Med Res. 2013 (51) \\
\hline & Simvastatina & $\begin{array}{l}\text { van den Akker JM, et al. J Nephrol. } 2003 \text { (56); } \\
\text { Shahbazian H, et al. Jundishapur J Nat Pharm Prod. } 2015 \text { (57) }\end{array}$ \\
\hline & Rosuvastatina & Tian J, et al. Ren Fail. 2017 (52) \\
\hline \multirow{5}{*}{$\begin{array}{l}\text { Bloccanti del sistema renina- } \\
\text { angiotensina-aldosterone }\end{array}$} & Ramipril & Gamboa JL, et al. BMC Nephrol. 2015 (58) \\
\hline & Enalapril & Ordaz-Medina SM, et al. ASAIO J. 2010 (59) \\
\hline & Losartan & Merino A, et al. Nephrol Dial Transplant. 2012 (60) \\
\hline & Olmesartan & Kadowaki D, et al. Hypertens Res. 2007 (61) \\
\hline & Aliskiren & Moriya H, et al. Kidney Blood Press Res. 2013 (62) \\
\hline \multirow{4}{*}{ Scelta del dialisato } & Ridotta conducibilità del sodio & Macunluoglu B, et al. Int Urol Nephrol. 2016 (69) \\
\hline & Con acido ascorbico & Shi XF, et al. Ren Fail. 2005 (70) \\
\hline & Con epigallocatechina gallato & Chen TS, et al. Food Chem. 2012 (71) \\
\hline & Con idrogeno molecolare $\mathrm{H} 2$ & Terawaki H, et al. Hemodial Int. 2014 (72) \\
\hline $\begin{array}{l}\text { Filtri ricoperti } \\
\text { di vitamina } \mathrm{E}\end{array}$ & & $\begin{array}{l}\text { Floridi A, et al. Acta Biomater. } 2009 \text { (73); } \\
\text { Yang SK, et al. Ren Fail. } 2014 \text { (74) }\end{array}$ \\
\hline $\begin{array}{l}\text { Utilizzo di eparina a basso peso } \\
\text { molecolare rispetto a eparina } \\
\text { non frazionata }\end{array}$ & & Poyrazoglu OK, et al. Ren Fail. 2006 (75) \\
\hline
\end{tabular}

re l'infiammazione nel dializzato, che sono sintetizzate nella Tabella I (34-74). Ricordiamo alcuni approcci che hanno deluso le aspettative: elevate dosi di tiamina e piridossina (76) e gli ACE inibitori. Infatti, la dimetilarginina asimmetrica (ADMA), che contribuisce alla disfunzione endoteliale nella
MRC avanzata, viene aumentata da ramipril ma non da valsartan (58). Inoltre, in un RCT in doppio cieco enalapril ha significativamente ridotto la pressione arteriosa ma non i livelli plasmatici di PCR e IL-6 rispetto al placebo (59).

La review qui presentata pone quindi l'accento sulle novi- 
tà più interessanti per combattere lo stress ossidativo e sulla necessità di traslare in trial clinici gli approcci della ricerca di base per colpire il processo infiammatorio, per migliorare la mortalità cardiovascolare del paziente dializzato.

\section{Disclosures}

Financial support: No financial support was received for this submission.

Conflict of interest: The authors have no conflict of interest.

\section{Bibliografia}

1. Ronco C, Levin NW. End-stage renal disease: a slowly progressive systemic inflammatory response syndrome. Contrib Nephrol. 2002;(137):379-85.

2. Zimmermann J, Herrlinger S, Pruy A, et al. Inflammation enhances cardiovascular risk and mortality in hemodialysis patients. Kidney Int. 1999;55:648.

3. Stenvinkel P. Inflammatory and atherosclerotic interactions in the depleted uremic patient. Blood Purif. 2001;19:53-61.

4. Santoro A, Mancini E. Cardiac effects of chronic inflammation in dialysis patients. Nephrol Dial Transplant. 2002;17(Suppl 8): S10-5.

5. Tonelli M, Karumanchi SA, Thadhani R. Epidemiology and mechanisms of uremia-related cardiovascular disease. Circulation. 2016;133:518-36.

6. Beddhu S, Kimmel PL, Ramkumar N, Cheung AK. Associations of metabolic syndrome with inflammation in CKD: Results from the third national health and nutrition examination survey (NHANES III). Am J Kidney Dis. 2005;46:577-86.

7. Baragetti I, El Essawy B, Fiorina P. Targeting Immunity in endstage renal disease. Am J Nephrol. 2017;45:310-9.

8. Himmelfarb J, Stenvinkel P, Ikizler TA, Hakim RM. The elephant in uremia: oxidant stress as a unifying concept of cardiovascular disease in uremia. Kidney Int. 2002;62(5):1524-38.

9. Li N, Karin M. Is NF-kappaB the sensor of oxidative stress? FASEB J. 1999;13:1137-43.

10. Vaziri ND, Dicus M, Ho ND, Boroujerdi-Rad L, Sindhu RK. Oxidative stress and dysregulation of superoxide dismutase and $\mathrm{NADPH}$ oxidase in renal insufficiency. Kidney Int. 2003;63:179-85.

11. Gollapudi P, Yoon JW, Gollapudi S, PahI MV, Vaziri ND. Leukocyte toll-like receptor expression in end-stage kidney disease. Am J Nephrol. 2010;31:247-54.

12. Liu G, Yang H. Modulation of macrophage activation and programming in immunity. J Cell Physiol. 2013;228:502-12.

13. Yoon JW, Pahl MV, Vaziri ND. Spontaneous leukocyte activation and oxygen-free radical generation in end-stage renal disease. Kidney Int. 2007;71(2):167-72.

14. Yamagishi S, Nakamura K, Matsui T, Noda Y, Imaizumi T. Receptor for advanced glycation end products (RAGE): a novel therapeutic target for diabetic vascular complication. Curr Pharm Des. 2008;14:487-95.

15. Baragetti I, Norata GD, Sarcina C, et al. -374 T/A RAGE polymorphism is associated with chronic kidney disease progression in subjects affected by nephrocardiovascular disease. PLoS One. 2013;8(4):e60089.

16. Guijarro C, Egido J. Transcription factor-kappa B (NF-kappa B) and renal disease. Kidney Int. 2001;59(2):415-24.

17. Ozkok A, Caliskan Y, Sakaci T, et al. Osteoprotegerin/RANKL axis and progression of coronary artery calcification in hemodialysis patients. Clin J Am Soc Nephrol. 2012;7(6):965-73.

18. Boaz M, Smetana $\mathrm{S}$, Weinstein $\mathrm{T}$, et al. Secondary prevention with antioxidants of cardiovascular disease in endstage renal disease (SPACE): randomised placebo-controlled trial. Lancet. 2000;356(9237):1213-8.

19. Gonzalez-Espinoza L, Rojas-Campos E, Medina-Pérez $M$, Peña-Quintero P, Gómez-Navarro B, Cueto-Manzano AM. Pentoxifylline decreases serum levels of tumor necrosis factor alpha, interleukin 6 and C-reactive protein in hemodialysis patients: results of a randomized double-blind, controlled clinical trial. Nephrol Dial Transplant. 2012;27(5):2023-8.

20. Heinz J, Kropf S, Domröse U, et al. B vitamins and the risk of total mortality and cardiovascular disease in end-stage renal disease: results of a randomized controlled trial. Circulation. 2010;121(12):1432-8.

21. Wu YQ, Qu H, Sfyroera G, et al. Protection of nonself surfaces from complement attack by factor $\mathrm{H}$-binding peptides: implications for therapeutic medicine. J Immunol. 2011;186: 4269-77.

22. Hezmee MN, Shiels IA, Rolfe BE, Mills PC. Complement C5a: impact on the field of veterinary medicine. Vet J. 2012;192: 264-71.

23. Kourtzelis I, Markiewski MM, Doumas M, et al. Complement anaphylatoxin C5a contributes to hemodialysis-associated thrombosis. Blood. 2010;116:631-9.

24. Hung AM, Ellis CD, Shintani A, Booker C, Ikizler TA. IL-1beta receptor antagonist reduces inflammation in hemodialysis patients. J Am Soc Nephrol. 2011;22(3):437-42.

25. Taki K, Takayama F, Niwa T. Beneficial effects of bifidobacteria in a gastroresistant seamless capsule on hyperhomocysteinemia in hemodialysis patients. J Ren Nutr. 2005;15:77-80.

26. Pletinck A, Glorieux G, Schepers E, et al. Protein-bound uremic toxins stimulate crosstalk between leukocytes and vessel wall. J Am Soc Nephrol. 2013;24:1981-94.

27. Litjens $\mathrm{NH}$, Boer K, Zuijderwijk JM, et al. Natural regulatory $\mathrm{T}$ cells from patients with end-stage renal disease can be used for large-scale generation of highly suppressive alloantigenspecific Tregs. Kidney Int. 2017;91(5):1203-13.

28. Casiraghi F, Perico N, Cortinovis M, Remuzzi G. Mesenchymal stromal cells in renal transplantation: opportunities and challenges. Nat Rev Nephrol. 2016;12(4):241-53.

29. Buccianti, G, RaselliS, Baragettil, et al.5-methyltetrahydrofolate restores endothelial function in uraemic patients on convective haemodialysis. Nephrol Dial Transplant. 2002;17(5):857-64.

30. van Guldener C, Janssen MJ, Lambert J, et al. No change in impaired endothelial function after long-term folic acid therapy of hyperhomocysteinaemia in haemodialysis patients. Nephrol Dial Transplant. 1998;13:106-12.

31. Nigwekar SU, Kang A, Zoungas $S$, et al. Interventions for lowering plasma homocysteine levels in dialysis patients. Cochrane Database Syst Rev. 2016;5:CD004683.

32. Don BR, Kim K, Li J, Dwyer T, Alexander F, Kaysen GA. The effect of etanercept on suppression of the systemic inflammatory response in chronic hemodialysis patients. Clin Nephrol. 2010;73:431-8.

33. Kay E, Scotland RS, Whiteford JR. Toll-like receptors: role in inflammation and therapeutic potential. Biofactors. 2014; 40:284-94.

34. Migliori M, Panichi V, de la Torre R, et al. Anti-inflammatory effect of white wine in CKD patients and healthy volunteers. Blood Purif. 2015;39(1-3):218-23.

35. Janiques AG, Leal Vde O, Stockler-Pinto MB, Moreira NX, Mafra D. Effects of grape powder supplementation on inflammatory and antioxidant markers in hemodialysis patients: a randomized double-blind study. J Bras Nefrol. 2014;36(4): 496-501.

36. Castilla P, Echarri R, Dávalos A, et al. Concentrated red grape 
juice exerts antioxidant, hypolipidemic, and antiinflammatory effects in both hemodialysis patients and healthy subjects. Am J Clin Nutr. 2006;84(1):252-62.

37. Wu PT, Fitschen PJ, Kistler BM, et al. Effects of Pomegranate extract supplementation on cardiovascular risk factors and physical function in hemodialysis patients. J Med Food. 2015;18(9):941-9.

38. Rassaf T, Rammos C, Hendgen-Cotta UB, et al. Vasculoprotective effects of dietary cocoa flavanols in patients on hemodialysis: a double-blind, randomized, placebo-controlled trial. Clin J Am Soc Nephrol. 2016;11(1):108-18.

39. Hsu SP, Wu MS, Yang CC, et al. Chronic green tea extract supplementation reduces hemodialysis-enhanced production of hydrogen peroxide and hypochlorous acid, atherosclerotic factors, and proinflammatory cytokines. Am J Clin Nutr. 2007; 86(5):1539-47.

40. Chen TS, Liou SY, Wu HC, et al. Efficacy of epigallocatechin-3gallate and Amla (Emblica officinalis) extract for the treatment of diabetic-uremic patients. J Med Food. 2011;14(7-8): 718-23.

41. Calo LA, Vertolli U, Davis PA, et al. Molecular biology based assessment of green tea effects on oxidative stress and cardiac remodelling in dialysis patients. Clin Nutr. 2014;33(3):437-42.

42. Shelmadine BD, Bowden RG, Moreillon JJ, et al. A Pilot Study to examine the effects of an anti-inflammatory supplement on eicosanoid derivatives in patients with chronic kidney disease. Clin Nutr. 2014;33(3):437-42.

43. Pu C, Yang YB, Sun QL. Effects of Salvia miltiorrhiza on oxidative stress and microinflammatory state in patients undergoing continuous hemodialysis. Zhongguo Zhong Xi Yi Jie He Za Zhi. 2006;26(9):791-4.

44. Qu XL, Dai Q, Qi YH, et al. Effects of Astragalous Injection on oxidative stress status in maintenance hemodialysis patients. Zhong Xi Yi Jie He Xue Bao. 2008;6(5):468-72.

45. Vernaglione L, Cristofano C, Chimienti S. Omega-3 polyunsaturated fatty acids and proxies of cardiovascular disease in hemodialysis: a prospective cohort study. J Nephrol. 2008;21(1):99-105.

46. Gharekhani A, Khatami MR, Dashti-Khavidaki S, et al. The effect of omega-3 fatty acids on depressive symptoms and inflammatory markers in maintenance hemodialysis patients: a randomized, placebo-controlled clinical trial. Eur J Clin Pharmacol. 2014;70(6):655-65.

47. Moeinzadeh F, Shahidi S, Mortazavi M, et al. Effects of Omega-3 fatty acid supplementation on serum biomarkers, inflammatory agents, and quality of life of patients on hemodialysis. Iran J Kidney Dis. 2016;10(6):381-7.

48. Chen Y, Abbate M, Tang L, et al. L-Carnitine supplementation for adults with end-stage kidney disease requiring maintenance hemodialysis: a systematic review and meta-analysis. Am J Clin Nutr. 2014;99(2):408-22.

49. Alshahawey M, Shahin SM, Elsaid TW, Sabri NA. Effect of Febuxostat on the endothelial dysfunction in hemodialysis patients: a randomized, placebo-controlled, double-blinded Study. Am J Nephrol. 2017;45(5):452-9.

50. Makówka A, Dryja P, Chwatko G, Bald E, Nowicki M. Treatment of chronic hemodialysis patients with low-dose fenofibrate effectively reduces plasma lipids and affects plasma redox status. Lipids Health Dis. 2012;11:47.

51. Cueto-Manzano AM, Angel-Zúñiga JR, Ornelas-Carrillo G, et al. Anti-inflammatory interventions in end-stage kidney disease: a randomized, double-blinded, controlled and crossover clinical trial on the use of pravastatin in continuous ambulatory peritoneal dialysis. Arch Med Res. 2013;44(8):633-7.

52. Tian J, Hou X, Hu L, et al. Efficacy comparison of atorvastatin versus rosuvastatin on blood lipid and microinflammatory state in maintenance hemodialysis patients. Ren Fail. 2017;39(1):153-8.

53. Stavroulopoulos A, Petras D, Kakavas I, et al. Monocyte expression of adhesion molecules during low- and highflux polysulfone hemodialysisand the effect of atorvastatin administration. Blood Purif. 2010;29(3):274-9.

54. Di Renzo L, Noce A, De Angelis S, et al. Anti-inflammatory effects of combined treatment with acetyl salicylic acid and atorvastatin in haemodialysis patients affected by Normal Weight Obese syndrome. Pharmacol Res. 2008;57(2):93-9.

55. Vernaglione L, Cristofano C, Muscogiuri P, Chimienti S. Does atorvastatin influence serum C-reactive protein levels in patients on long-term hemodialysis? Am J Kidney Dis. 2004;43(3):471-8.

56. van den Akker JM, Bredie SJ, Diepenveen SH, et al. Atorvastatin and simvastatin in patients on hemodialysis: effects on lipoproteins, C-reactive protein and in vivo oxidized LDL. J Nephrol. 2003;16(2):238-44.

57. Shahbazian H, Atrian A, Yazdanpanah L, Lashkarara GR, Zafar Mohtashami A. Anti-inflammatory effect of simvastatin in hemodialysis patients. Jundishapur J Nat Pharm Prod. 2015;10(1):e17962.

58. Gamboa JL, Pretorius M, Sprinkel KC, Brown NJ, Ikizler TA. Angiotensin converting enzyme inhibition increases ADMA concentration in patients on maintenance hemodialysis--a randomized cross-over study. BMC Nephrol. 2015;16:167.

59. Ordaz-Medina SM, González-Plascencia J, Martín del Campo $F$, et al. Is systemic inflammation of hemodialysis patients improved with the use of enalapril? Results of a randomized, double-blinded, placebo-controlled clinical trial. ASAIO J. 2010;56(1):37-41.

60. Merino A, Alvarez-Lara MA, Ramirez R, et al. Losartan prevents the development of the pro-inflammatory monocytes CD14+CD16+ in haemodialysis patients. Nephrol Dial Transplant. 2012;27(7):2907-12.

61. Kadowaki D, Anraku M, Tasaki Y, et al. Effect of olmesartan on oxidative stress in hemodialysis patients. Hypertens Res. 2007;30(5):395-402.

62. Moriya H, Kobayashi S, Ohtake T, et al. Aliskiren, a direct renin inhibitor, improves vascular endothelial function in patients on hemodialysis independent of antihypertensive effect, a pilot study. Kidney Blood Press Res. 2013;37(2-3):190-8.

63. Rodríguez-Osorio L, Zambrano DP, Gracia-Iguacel C, et al. Use of sevelamer in chronic kidney disease: beyond phosphorus control. Nefrologia. 2015;35(2):207-17.

64. Choi SR, Lim JH, Kim MY, et al. Cinacalcet improves endothelial dysfunction and cardiac hypertrophy in patients on hemodialysis with secondary hyperparathyroidism. Nephron Clin Pract. 2012;122(1-2):1-8.

65. Nakamura $\mathrm{Y}$, Tsuji $\mathrm{M}$, Hasegawa $\mathrm{H}$, et al. Anti-inflammatory effects of linagliptin in hemodialysis patients with diabetes. Hemodial Int. 2014;18(2):433-42.

66. Izuhara Y, Miyata T, Saito K, et al. Ultrapure dialysate decreases plasma pentosidine, a marker of "carbonyl stress". Am J Kidney Dis. 2004;43(6):1024-9.

67. Honda $H$, Suzuki $H$, Hosaka $N$, et al. Ultrapure dialysate influences serum myeloperoxidase levels and lipid metabolism. Blood Purif. 2009;28(1):29-39.

68. Susantitaphong P, Riella C, Jaber BL. Effect of ultrapure dialysate on markers of inflammation, oxidative stress, nutrition and anemia parameters: a meta-analysis. Nephrol Dial Transplant. 2013;28(2):438-46

69. Macunluoglu B, Gumrukcuoglu HA, Atakan A, et al. Lowering dialysate sodium improves systemic oxidative stress in maintenance hemodialysis patients. Int Urol Nephrol. 
2016;48(10):1699-704.

70. Shi XF, Ding F, Zhu QY, et al. Use of ascorbate-rich dialysate to attenuate oxidative stress in maintenance hemodialysis patients. Ren Fail. 2005;27(2):213-9.

71. Chen TS, Liou SY, Wu HC, et al. The application of (-)-epigallocatechin gallate in preparation of an antioxidant dialysate. Food Chem. 2012;134(3):1307-11.

72. Terawaki H, Zhu WJ, Matsuyama Y, et al. Effect of a hydrogen $(\mathrm{H} 2)$-enriched solution on the albumin redox of hemodialysis patients. Hemodial Int. 2014;18(2):459-66.

73. Floridi A, Piroddi M, Pilolli F, et al. Analysis method and characterization of the antioxidant capacity of vitamin E-interactive polysulfone hemodialyzers. Acta Biomater. 2009;5(8):2974-82.
74. Yang SK, Xiao L, Xu B, et al. Effects of vitamin E-coated dialyzer on oxidative stress and inflammation status in hemodialysis patients: a systematic review and meta-analysis. Ren Fail. 2014;36(5):722-31.

75. Poyrazoglu OK, Dogukan A, Yalniz M, Seckin D, Gunal AL. Acute effect of standard heparin versus low molecular weight heparin on oxidative stress and inflammation in hemodialysis patients. Ren Fail. 2006;28(8):723-7.

76. Nascimento MM, Suliman ME, Murayama $Y$, et al. Effect of high-dose thiamine and pyridoxine on advanced glycation end products and other oxidative stress markers in hemodialysis patients: a randomized placebo-controlled study. J Ren Nutr. 2006;16(2):119-24. 